\title{
A Wireless Solution to Collecting and Displaying Oil Temperature Data Based on Zigbee Network
}

\author{
Cui Jingcong ${ }^{\text {a1 }}$, Chen Lidong ${ }^{\mathrm{a}}$,Li Xunming ${ }^{\mathrm{a} 2}$,Huang Chunhai ${ }^{\mathrm{b}}$ \\ ${ }^{a}$ Department of Energy and Electrical Engineering, Hohai University Nanjing,210036,China \\ ${ }^{b}$ Nanda Digital Science \& Technology CO. Nanjing, China
}

\begin{abstract}
Abstract - In some oil fields of China, monitoring oil temperature during producing almost relies on manual operation. According to this, we designed a solution based on Zigbee network and sensor technology. In our design of sampling nodes, with JN5139 module as the core, uses sensor PT100 to collect temperature data and LCD module LCM141C-01 to display the final result. The whole solution has an advantage of a simple structure, low power consumption etc, therefore, it has a positive meaning in improving monitoring work efficiency in oil fields.
\end{abstract}

Index Terms: Oil Temperature Monitoring,Zigbee, JN5139, PT100, LCM141C-01

(C) 2012 Published by MECS Publisher. Selection and/or peer review under responsibility of the Research Association of Modern Education and Computer Science

\section{Introduction}

In recent years, the degree of domestic oil fields' automation keeps increasing. The application of various new technical and automate equipments in monitoring plays an important role in guaranteeing safe and efficient production in oil fields. In the link of monitoring oil well-pumping, the temperature of the oil should be monitored in order to avoid the condensation of crude oil, which may negatively influence the normal oil pumping. Oil temperature monitoring work is usually carried out by staff when inspecting the pumping well. They measure the temperature of the oil tube wall with the help of mercury thermometer. Temperature monitor measurement like this could not get precise results and carries great limitations. It could not cover the numerous oil wells in discrete locations and complicated environments. With the reality and the complicated industrial environment of oil fileds considered, it demands a wireless solution of lower complexity, power consumption, cost and rate but anti-interference. The $2.4 \mathrm{GHz}$ Zigbee-based display system for oil temperature data collection mentioned below could well solve the above problems.

\footnotetext{
* Corresponding author.

E-mail address: ${ }^{1}$ cuijingcon@163.com ${ }^{2}$ 1xm@vip.sina.com
} 


\section{Design Overview}

The design mainly consists of the Zigbee wireless network nodes and the upper computer (PC). According to the different roles in network, wireless network nodes could be classified into three types: end device, router and coordinator. The structure of the whole system is shown in Fig.1.

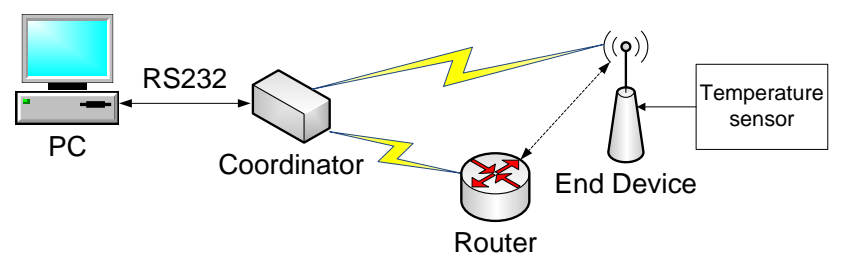

Fig. 1 Design Overview

End device(namely sampling node),powered by rechargeable lithium batteries, is installed on the pipe under the beam pumping unit. The node's metal pole with temperature sensors inside goes deep into the oil pipe, and is tangent to the surface of the liquid oil. The major tasks for end device include: collecting oil temperature at setting time interval, saving reasonable data into Flash and displaying them through its LCD monitor; receiving and responding to the commands from upper computer, sending data back to the PC in control room through wireless network. End device usually stays in sleeping state while free to reduce power consumption. Router plays a pivotal role in the network, i.e. forwarding the command from coordinator or data from end device. It could also communicate with other routers. Coordinator is the core of the whole network, responsible for the starting the network, communicating with and managing child nodes. In control room, Coordinator usually connects and interacts with upper computer through serial port.All Zigbee networks must have one(and only one)coordinator, irrespective of the network topology[1].

\section{Hardware Design}

The network nodes take the module JN5139-Z01-Mxx, produced by Jennic Corporation, as the core and low power consumption as the selection for device selection. Take the end device, which is most complicated in structure, shown as an example in Fig.2.

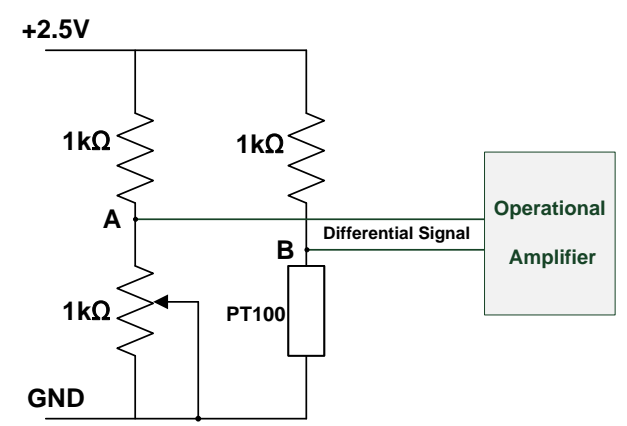

Fig. 2 Hardware Design of End Device

End device collects temperature through temperature sensor. The sampling signals will be used as the input of JN5139 module's ADC pin. JN5139 module will process the A/D converted digital signal and read the records of sampling time from clock module, then save the data into the flash, transmit them to the LCD monitor through 
SPI(Serial Peripheral Interface) and display the result. The power module includes a rechargeable lithium battery and voltage regulator chip. It's also designed with the solar panel as the power supplement. The clock module adopts dual power mode, i.e. when end device is powered, the clock module is charged with mains; when the outer mains is off, it's charged by the backup battery.

\section{A. Module JN5139}

Module JN5139-Z01-Mxx integrates the JN5139 chip, 128KB Flash and built-in antenna interface. The point-to-point stable transmission distance reaches to $200 \mathrm{~m}$. JN5139 is a low power consumption and high efficient wireless microcontroller chip, integrated with 32-bit RISC processor. $2.4 \mathrm{GHz}$ sending and receiving module. The chip is equipped with rich digital and analog peripheral interfaces. The transceiver integrated in JN5139 is processed with 128-bit AES encryption, CRC checking mechanism[2]. So whatever transmission security or anti-interference, module JN5139 is stronger than the traditional $433 \mathrm{MHz}$ wireless communication device. And take the consideration of power consumption, the currency is only $34 \mathrm{~mA}$ under normal sending and receiving state, in sleep mode it can be as low as $\mu \mathrm{A}$-level.

\section{B. Temperature Sensor}

End device adopts PT100 platinum temperature sensor to collect temperature data. PT100 temperature sensor could stand high pressure and vibration and have more stability and accuracy. Its measure scale ranges from -200 $-850^{\circ} \mathrm{C}[3]$. The two ends of PT100 could be connected to the bridge circuit. The temperature collecting circuit just as the Fig. 3 shows.

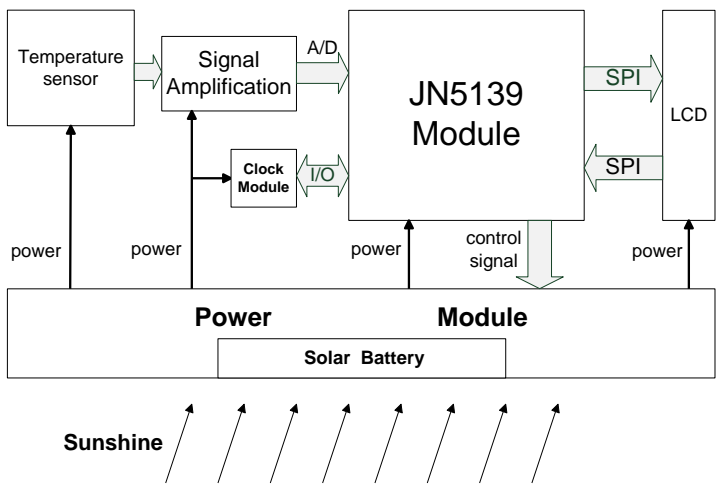

Fig.3 PT100 Bridge Circuit

Both ends of the bridge temperature-measure circuit are protected by TL431 with the stable voltage $2.5 \mathrm{~V}$. The differential signal output from bridge A and B is amplified by the operational amplifier. The voltage obtained ranges from 0 to $2.4 \mathrm{~V}$, i.e. within the allowed range of the JN5139 chip ADC pin.

\section{LCD Module}

To facilitate the field staff to monitor the real-time oil temperature when on patrolling, end device is designed with LCD module to show the oil temperature data. The LCD monitor selects 14-bit, 8-segment of the module LCM141C-01 produced in China. The typical value of the currency under LCM141C-01 display state is only $4 \mu \mathrm{A}$ and less than $1 \mu \mathrm{A}$ under the power-saving state. Its micro-power consumption is very significant. LCM 141C-01also has the advantages of being stable, simple programmed and suitable as a measuring instrument-specific LCD module[4].

LCM141C-01 integrated with driver and control circuit, simply interfaced. It could communicate with the module JN5139 through SPI. Its real-time signal could be provided by internal and external crystal oscillator 
(with $32.768 \mathrm{KHz}$ crystal welding outside the module). The design of end device adopts the internal oscillation mode.

\section{Data Processing and Programming}

\section{A. Processing Temperature Data}

Well considering the actual oil temperature is in the range of $0-100{ }^{\circ} \mathrm{C}$, find type value table of PT100 thermal resistance in the range. With the help of Matlab, use these discrete points in reference table[5]to do quadratic curve fitting. Before and after curve fitting, draw diagram of PT100 platinum resistance and the relation of temperature as shown in Fig. 4

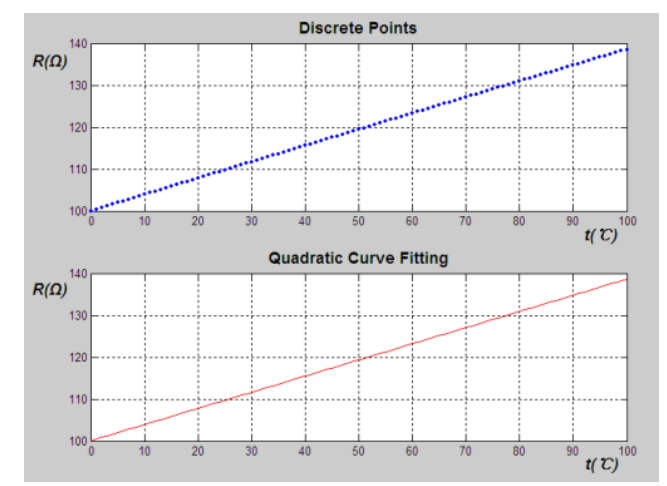

Fig. 4 R-t Diagram before\&after Curve Fitting in $0-100^{\circ} \mathrm{C}$

It can be observed that within the range of $0-100^{\circ} \mathrm{C}$, the resistance of PT100 is high in linearity. On the premise of less precision demand on monitoring, R-t could be approximately regarded as linear.

Get quadratic polynomial:

$$
R=A^{2}+B t
$$

Where constants:

$$
A=-5.7645 * 10^{-5} \quad B=3.9086 \quad C=99.999
$$

As the gain of the operational amplifier is known, measure JN5139 12-bit (digital value $0 \sim 4095$ ) ADC pin maximum voltage input Vmax. Vmax corresponds to 4095, namely obtain the linear relationship between analog and digital value. Therefore, when having gotten the digital value, voltage between $\mathrm{A}$ and $\mathrm{B}$ can be calculated. Then use the circuit knowledge and R-t relation, calculate the temperature value in range $0-100{ }^{\circ} \mathrm{C}$

\section{B. Programming for Nodes}

Programming is designed under the platform of Code::Blocks based on the protocol of $2.4 \mathrm{GHz}$ Zigbee provided by Jennic corporation. The application program of node is to operate on the CPU and peripheral devices through BOS and Zigbee protocol stack. BOS (Basic Operating System) is actually a simple task scheduler. Take end device as an example, the main program flow diagram is shown in Fig. 5

Since the design uses a beacon network form, i.e., in the case that child nodes didn't manage to join a network, they would automatically scan channels and try to find a coordinator by sending beacon requests to join the network. To reduce power consumption of end device, make the device sleep 10 seconds when it hasn't joined a network. When end device managed to join one, the sleeping time is designed much less, as 2seconds. It's 
because end device may have to receive and respond to commands from its parent node. End device processes commands with the event trigger mechanism.

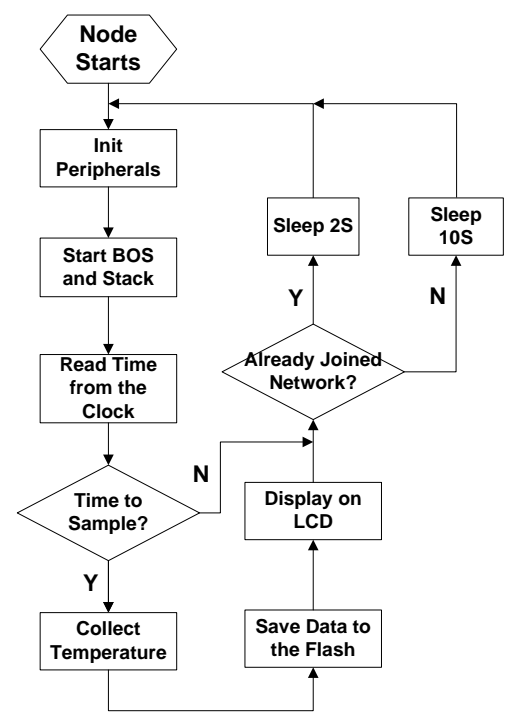

Fig .5 Main Program Flow of End Device

\section{Programming for Upper Computer}

Upper computer (PC) is connected via serial port with coordinator, its main functions are: writing commands to the serial port at setting time; receiving data from COM port and making analysis; displaying results for administrator users. The flow chart is shown in Fig.6

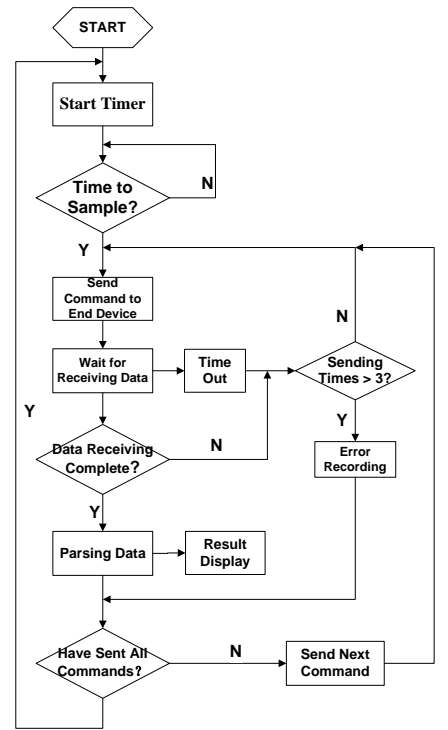

Fig.6 Main Program Flow of Upper Computer 


\section{Conclusion}

The solution based on Zigbee wireless network is simple in structure, low in power consumption and has more superior performances than $433 \mathrm{MHz}$ wireless solution. The design could not only be applied to measure and display temperature of liquid like crude oil, but also be utilized as a reference in the automatic monitoring filed like building temperature monitoring. It is believed that with the continuous improvement of WSN (wireless sensor network ) technology, more similar Zigbee wireless solutions would turn up. And they would have greater advantage not only in costs but also in technology, play a more important role in promoting production and improving people's livings.

\section{ACKNOWLEDGMENT}

Project Supported by National Science Foundation of China (60874013)

\section{References}

[1] JN - UG - 3017 - ZigBeeStackUserGuide. Useer Guide for JN5139, Jennic Corporation, Version:1v6

[2] JN - DS - JN513x - 1v4. Data Sheet for JN5139, Jennic Corporation

[3] Zhiwen Chen, Wei Wang. Design and Realization of Temperature Transmitter Based on Pt100 Platinum Thermal Resistance. Modern Electronics Technique[J], 2010,8 : P197 - 199(in Chinese)

[4] PT100 Platinum Temperature Sensor Manul. Yiyuetong-Electronic Corporation,Beijing,China(in Chinese)

[5] Weigang Zhang, Jing Zheng. The LCD - LCM141 and Its Application. International Electronic Elements[J], June,2003:P51 - 54 (in Chinese) 